\title{
TECHNOLOGIES FOR TRAINING AND INTENTIONAL STANCE
}

\author{
Oronzo Parlangeli ${ }^{1}$ and Paul M. Liston ${ }^{2}$ \\ ${ }^{1}$ Dipartimento di Scienze Sociali, Politiche e Cognitive, Università di Siena, Siena, Italy \\ ${ }^{2}$ Centre for Innovative Human Systems, Trinity College Dublin, The University of Dublin, Dublin, Ireland
}

\begin{abstract}
Interactions with digital technologies can be based on the adoption of relational strategies which assume that these technologies have mental states. There are at least two reasons to support the hypothesis that intentional stance is the priority relational strategy. First, the evolutionary path that, at an individual level, leads us to take on a design stance towards artifacts and which appears slower, and later, than that relating to the intentional stance. Second, the fact that the recognition of interactive digital technologies as simple artifacts is something that requires commitment and is not immediate: when overloaded we can slide from design stance towards intentional stance.
\end{abstract}

\section{KEYWORDS}

Mental States, Theory of Mind, Relational Strategies, Digital Technologies

\section{REFLECTION PAPER}

Several studies highlight the ways in which interactions with digital technologies can be based on the adoption of mental models and relational strategies which assume that these technologies have mental states, or intentions. It seems that, at least in some circumstances, when we are dealing with digital technologies we sometimes implement interactive behaviors similar to those that occur between human beings (Parlangeli, Guidi \& Fiore Farina, 2012; Thellman, Silvervarg, \& Ziemke, 2017; Marchesi et al., 2019; Perez-Osorio \& Wykowska, 2020).

Daniel Dennett $(1971 ; 1987)$ advanced a theoretical framework which can be considered a point of reference, and which proposes three interpretative strategies of the world - the physical stance, the design stance and the intentional stance. The behavioral predictions of entities such as stones or stars are essentially based on the adoption of a physical stance. When, on the other hand, we enter into a relationship with artificial systems, the functional aspects become dominant and therefore relational strategies should be based on the use of the design stance. Finally, with regards to humans the predictions of behavior are based on the thoughts of others, their intentions, beliefs, and mental states, and in these cases we adopt an intentional stance. Dennett proposed that the assumption of one or other stance is due to the suggestions that the systems provide us directly and to the evolution of the interaction with them. However, the consideration that the intentional stance must refer to the capability to elaborate a Theory of Mind -ToM - (Baron Cohen, 1995) seems to suggest that intentional stance is the most complex and advanced form of interaction, and therefore the last to come into play both in the course of individual psychological development and in the evolution of interactions with the world

Nonetheless, various studies indicate the intentional stance as the basis of the possibility of establishing social and cognitive development, and it is thanks to this that various forms of cultural learning can be achieved (Tomasello et al., 2005; Perez-Osorio \& Wykowska, 2020). And the elaboration of a thought which reduces artificial interactive systems to the rank of mere artifacts is probably the result of a path that develops more slowly, the result of our cultural evolution that allows us to slowly discriminate that which is natural from that which is not in a given context. In short, we can advance that the basic strategy in establishing a relationship with the world is that which foresees the supposition of a causative mechanism of behavior which is comparable to a mind. There are at least two reasons to support the hypothesis that intentional stance is the priority relational strategy with the world. 
The first has to do with the evolutionary path that, at an individual level, leads us to take on a design stance towards artifacts and which appears slower, and later, than that relating to the intentional stance. Babies as young as 5 months-old already adopt initial forms of intentional stance since they are shown to be able to follow the gaze of adult agents on a screen (Senju, Csibra, \& Johnson, 2008). When they are nine months old they understand that actions have goals and that if these goals are achieved then emotional states will emerge, such as happiness for example, that are different from those related to failure to achieve the goals (Tomasello et al., 2005). If we talk about design stance, however, the developmental path is probably different and slower. Indeed, children are shown to be sensitive to certain types of functional affordances of objects only after they are 12 months-old. And it's only after they are 24 months-old that they are able to attribute a sole function to an object, usually that demonstrated by an adult (Kelemen \& Carey, 2007). From 4-6 years of age children start to have an ability to determine the category of an object according to the designer's intentions and the evolution of its use.

The second reason, refers to the fact that strategies of interaction with artificial systems can change according to the cognitive commitment in the execution of some tasks. In fact, some research has shown that participants who are induced to operate in a multitasking mode during the execution of a task, and consequently can devote less cognitive resources to the interpretation of the system with which they interact, are more likely to use intentional stance, and therefore to attribute mental states to this system (Parlangeli, Guidi, \& Fiore Farina, 2012; Parlangeli, Caratozzolo \& Guidi, 2014). It's as if the correct recognition of interactive digital technologies as simple artifacts is something that requires commitment, procedural resources on a cognitive level that, if removed, make the relationship slide towards less burdensome, more immediate modes, those that are based on the adoption of intentional stance. Relations with technology seem to be prioritising, you might say privileging, the adoption of intentional stance.

In the case of educational technologies this could have considerable relevance, it could completely change the underlying ideas about their design as well as the training aimed at their use. Maybe, for example, it is time to consider that a child who, for educational purposes, cooperates with a social robot will be led to think that it is equipped with a mind and, as such, capable of forming a relationship that provides mental states able to elaborate recursive and reflexive cognitive processes - even if robots were perhaps not designed precisely with these capabilities. Consequently, this would bring about implications for the training of designers and developers of education systems - competence development would need to include skills and techniques on the development and fostering of relationships among peers.

\section{REFERENCES}

Baron Cohen, S., 1995. Mindblindness. Learning, development, and conceptual change. MIT Press: Cambridge, MA.

Dennett, D.C., 1971. Intentional systems. Journal of Philosophy, 68 (February), 87- 106.

Dennett, D.C., 1987. The intentional stance. MIT Press: Cambridge, MA.

Kelemen, D. and Carey, S., 2007. The essence of artifacts: Developing the design stance. In S. Laurence and E. Margolis (eds) Creations of the mind: Artifacts and their representation. Oxford University Press, Oxford.

Marchesi, S., Ghiglino, D., Ciardo, F., Perez-Osorio, J., Baykara, E., and Wykowska, A., 2019. Do We Adopt the Intentional Stance Toward Humanoid Robots?. Frontiers in psychology, 10, 450.

Parlangeli, O., Chiantini, T. and Guidi, S., 2012. A Mind in a disk: The attribution of mental state to technological systems. Work, 41, 1118-1123.

Parlangeli, O., Guidi, S. and Fiore Farina, R., 2012. Overloading Disks onto a Mind: Quantity Effects in the Attribution of Mental States to Technological Systems. Proceedings of the 18th international Congress, IEA - International Ergonomics Association, Recife, 12-16 February, 2012.

Parlangeli, O., Caratozzolo, M.C., and Guidi, S., 2014. Multitasking and Mentalizing Machines: How the Workload Can Have Influence on the System Comprehension 11th International Conference, EPCE 2014, Held as Part of HCI International 2014, Heraklion, Crete, Greece, June 22-27, 2014, 50-58.

Perez-Osorio, J. and Wykowska, A., 2020. Adopting the intentional stance toward natural and artificial agents, Philosophical Psychology, 33: 3, 369-395.

Senju, A., Csibra, G., and Johnson, M.H., 2008. Understanding the referential nature of looking: Infants' preference for object-directed gaze. Cognition, 108, 2, 303-319.

Thellman, S., Silvervarg, A., and Ziemke, T., 2017. Folk-psychological interpretation of human vs. humanoid robot behavior: Exploring the intentional stance toward robots. Frontiers in Psychology, 8 (NOV), 1- 14.

Tomasello, M., Carpenter, M., Call, J., Behne, T., and Moll, H., 2005. Understanding and sharing intentions: The origins of cultural cognition. Behavioral and Brain Sciences, 28, 675- 691. 